\title{
What Brazil is doing to promote healthy diets and active lifestyles
}

\author{
Denise Coitinho ${ }^{1, *}$, Carlos A Monteiro ${ }^{2}$ and Barry M Popkin ${ }^{3}$ \\ ${ }^{1}$ Food and Nutrition Policy Unit, Ministry of Health, SHIS QI 21, Conjunto 06, Casa 17, CEP 71655-260, Brasilia, \\ Brazil: ${ }^{2}$ São Paulo University, Center for Epidemiological Studies in Health and Nutrition (NUPENS/USP), Av. Dr \\ Arnaldo 715, São Paulo 01246-904, SP-Brazil: ${ }^{3}$ School of Public Health, Carolina Population Center, CB \# 8120 \\ University Square, University of North Carolina at Chapel Hill, Chapel Hill, NC 27516-3997, USA
}

\begin{abstract}
Objectives: To present the way the Brazilian government is addressing the prevention of nutrition-related non-communicable diseases (NR-NCDs).

Results: Innovative legislative and regulatory actions, mass communications and capacity building have been combined to create a comprehensive approach for addressing poor dietary and activity patterns in Brazil that are leading to obesity and NR-NCDs. Included are new nutrition-related initiatives in the labelling area, shifts in the types of food purchased for the school food programme, use of mass media to communicate components of the food guidelines, establishment of a smart shopping initiative, and training of teachers and health workers.

Conclusions: The entire effort has taken several years to get underway. This paper describes the process and some of the initial changes seen.
\end{abstract}

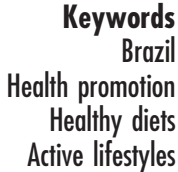

Changes in the nutritional status of the Brazilian population have been intense in the last quarter of the 20th century $^{1,2}$. Although undernutrition systematically declined from 1975 to 1997 for all ages, regions and income groups, nutritional deficiencies are still important in the country, particularly among lower-income children living in the north-eastern region. Obesity has increased, in the same time period, throughout the country among older children, adolescents and adults, with the single exception of middle- and higher-income, adult women in the south-eastern region - for whom the trends towards increased obesity seem to have stopped or even reversed in the 1990s. Undernutrition largely predominated over obesity in all regions and age groups of the Brazilian population in 1975, while in 1997 , the same picture was only seen among lower-income, younger children. Evidence of simultaneous changes towards high energydense diets and lower energy expenditure patterns was also documented across the country, as well as the increasing importance of diet- and sedentary-lifestylerelated non-communicable diseases.

To cope with these rapid changes, the Brazilian government has been working at the national and local level to initiate a wide range of activities to address these emerging needs. A new national food and nutrition policy has been developed following a wide-ranging set of discussions, meetings and negotiations between the Ministry of Health, relevant parts of Brazilian civil society (scholars, professional and scientific associations, workers' unions, representatives of private companies, and others) and other relevant governmental bodies (including those linked to the economic, agricultural, education and law sectors) ${ }^{3}$. The main goal of the new policy is essentially to promote, protect and support eating practices and lifestyles conducive to optimum nutritional and health status for all.

This new policy attempts to continue to combat nutritional deficiencies with focused and targeted integrated interventions. It also attempts to shift increasing attention towards the prevention of nutritional disorders associated with over- or wrong feeding and sedentary lifestyles. These actions and programmes focus on largescale activities aimed at behavioural change to create healthier eating and increased physical activity patterns. To reach that goal, strategies were conceptualised within a framework that includes three broad-linked areas of work: legislation, information/communication, and capacity building. The settings include broad-based social communication campaigns, as well as more focused information/communication interventions at schools, workplaces and in the health services. These are accompanied by interventions aimed at capacity building for schoolteachers and health workers and also by more fundamental legislative measures, without which the delivery of information/communication to the general public or to specific populations would not find coherent social environments to sustain behavioural change. The following section provides examples of some of the more exemplary activities in the three areas of work. Most are nation-wide initiatives, but some city- or state-level initiatives are also included since they have great potential to be implemented throughout the country. 


\section{Examples of important or innovative activities}

Two examples of innovative activities carried out in each of the three areas of work - legislation, information/ communication and capacity building - are given for illustrative purposes. More of the programmes that are underway or will be initiated during 2001/02 in Brazil can be found on the Brazilian Ministry of Health's website (www.saude.gov.br) ${ }^{3}$.

\section{Legislative and regulatory actions}

\section{Nutrition labelling and nutrition claims regulations}

Key components. Legislative action mandated that all packaged foods in Brazil list their content in calories, protein, carbohydrates, total fats, saturated fats, cholesterol, calcium, iron, sodium and dietary fibre in standardised, consumer-friendly tables. The information will be presented by serving sizes and percentage of recommended daily quantities. In parallel to this resolution, Brazilian serving sizes were established and introduced, for the first time in the world, the concept of 'nutritionally adequate serving sizes'. These serving sizes not only represent the usual consumption of different foods, but also provide a means of reinforcing unbalanced and erroneous eating patterns. They were, therefore, corrected for a nutritionally balanced diet. The information displayed on the food labels can be derived from the Brazilian food composition tables or direct analysis. A new food composition table, commissioned by the Ministry of Health to be developed by the University of Campinas, will include 250 foods analysed for micronutrients, and will be available very soon through the Internet. The resolution also established the need to have information on the nutritional content of raw and unpacked foods at point of sale.

Also, as part of the effort to better inform consumers, the Ministry of Health is reviewing the regulation on nutrition and health claims as well as the legislation relative to food advertisements directed to children. A mandatory nutrition labelling policy in both these efforts is a first and essential step, as its information comprises the basis of such complementary legislation.

Process of achieving consensus. This packaged-food information regulation process was constructed with intense participation of the Brazilian academic community and the food industry in a series of workshops. Once the technical basis for establishing the serving sizes was determined by the Ministry of Health and a draft of the regulation was presented, the food industry participants identified the serving size for each type of packaged food product available on the Brazilian market. It was one of the most participatory and co-operative processes involving government and the private sector ever accomplished regarding food and nutrition. The initial resistance by the Brazilian food industry was gradually overcome by this group work exercise, and their representatives became the best allies in the fight against nutritionally inadequate serving sizes.

A draft of the resolution and the serving sizes was then available on the Internet for 40 days, to allow anyone, who wanted to do so, an opportunity to make further suggestions. The Ministry of Health approved a resolution on 21 March 2001 for the mandatory nutrition labelling of all processed foods including the Brazilian serving sizes ${ }^{4}$.

Progress to date. Is the Brazilian consumer able to read, interpret and use the nutrition information available on the food labels? To answer that question, the Ministry of Health conducted surveys both in the toll-free telephone information service (about 6000 calls were received by the service during one week) and at several points of sale in the city of Brasilia. The new food label was also pre-tested in a variety of focal groups from different socio-economic backgrounds. As a general conclusion, it was found that consumers want to have that information available, whether they use it or not in the present format; want food labels to be more 'prescriptive' in terms of nutrition information; and want more advice on what to eat and what to avoid. They urged that labels provide nutrition claims or other consumer-friendly 'translation' of the nutrition information. Therefore, a whole communication strategy is being designed to provide further information to consumers on the nutrition information presented on the new food label.

The commercial media has also engaged in this process. There is a time/space dedicated to 'services to the public' in each media vehicle (newspapers, magazines, television programmes). Food and nutrition are topics highest in the public preference. This makes it feasible for health authorities to create strong partnerships with the commercial media sector to deliver nutrition messages and dietary guidelines. The nutrition labelling and serving sizes have received extensive attention in Brazil's commercial media, a critical component of our dissemination strategy.

The experience to date has shown that although there may be a lengthy time for all Brazilian consumers to make best possible use of the nutrition information, mandatory nutrition labelling has already served important purposes:

- it fulfils consumers' rights to information and consumer protection associations' goals. It has led, therefore, to important partnerships with organised consumer groups for the promotion of nutrition through their communication channels;

- it has placed nutrition promotion on the national agenda, due to the large coverage by the commercial media sector;

- it has enhanced government and food industry dialogue concerning the promotion of nutrition; and

- it is a first and mandatory step for the establishment of nutrition claims, codes for the marketing of 
industrialised foods or other forms of providing nutrition information on food labels, allowing citizens to make better food choices.

\section{Legislation to promote bealthy diets in schools}

Key components. Schools are, without question, one of the most important settings in which to carry out communication activities to promote healthy eating for a number of reasons: accessibility to ideal age groups, frequency of contacts, availability of various strategies that can be used and possibilities of creating group behaviour, among others.

In Brazil however, as in a number of countries, there is a fundamental contradiction within schools concerning the quality of school meals, the foods commercialised in school canteens and educational messages delivered to the students.

In 2000, new legislation was established in Brazil as an important step towards providing better school meals to elementary public school students (more than 37 million children) and towards obtaining better coherence within the school setting between educational messages about food habits and school meals. The federal government, through monthly monetary transfers to municipal authorities or directly to schools, funds the school meal programme. Food purchases are made locally. Brazil has 5561 municipalities throughout the country. According to the new legislation, it is mandatory that a minimum of $70 \%$ of the programme's annual budget of about US $\$ 500$ million is spent on fresh vegetables, fruits and minimally processed foods, preferably purchased from local producers and cooperatives of small farmers. The local authorities had been giving priority to the purchase of pre-cooked industrial foods for allegedly practical reasons, as they claimed that proper preparation of meals in the school setting required more human resources. To overcome this, efforts are being made, and resources are being allocated, for local producers and local farmers to pre-prepare (clean, chop, etc.) fresh foods for school meals in micro-scale agroindustries. Apart from improving the school children's diets, this is also a means to create a market share for smallscale agriculture with aggregate value. Foods sold in public school canteens are also being studied and probably will be regulated in the near future.

Progress to date. There has not been time to undertake any major impact evaluation. Brazilian municipalities and schools are adjusting their purchasing procedures according to the new regulation, and one of the main challenges is capacity building of the food preparers, called 'merendeiras', at the school level. These are usually low educated women. Some interesting examples can already be seen. In some municipalities of São Paulo State, a capacity-building project in partnership with local top restaurant chefs is taking place as an example of the array of possibilities to involve civil society in the improvement of nutrition.

\section{Information/communication activities}

\section{How to communicate the food guidelines?}

Key concepts. Nutrition experts wish to deliver several messages simultaneously in communication activities. All food guidelines have at least 10 'steps' or 10 'master messages' about fruits and vegetables, fats, salt, alcohol, water, physical activity, and so forth. Our experience in Brazil has shown that too many messages at one time is equal to no message being apprehended by the public; 10 messages are considered ineffective.

Brazil has produced, as have many other countries, the Brazilian Food Guidelines with the support of the Brazilian academic community. These guidelines are food-based and quantitative (as much as possible) to be concrete and feasible to be followed. One set of 10 guidelines is devoted to children up to two years of age and comprises messages to promote breast-feeding and sound, culturally specific and timely complementary feeding ${ }^{5}$. Another set of 10 guidelines is devoted to the general population using international recommendations with appropriate local adaptations. Two of the 10 steps refer to the need for increasing physical activity.

The innovation introduced in this work was the concept of a 'step-by-step route to healthy eating'. Instead of delivering 10 messages at once to the public, it was proposed that people choose one of the 10 recommended steps and pursue it as a personal or family goal. The 10 steps are logically ordered; therefore the majority of the population will probably pick step one. Whenever that initial goal is reached, it is proposed that people re-analyse the ' 10 steps' to choose another goal. It is likely that the accomplishment of one goal has also led to broader dietary changes and people will find that other steps have already been accomplished.

As a helpful tool for this process, we have produced and pre-tested a simple self-assessment questionnaire to evaluate one's diet. The test grade indicates the step to be taken first. More than 10 million copies of the selfassessment questionnaire and additional information on the 'step-by-step route to healthy eating' will be distributed to Brazilian adults in November 2001 during the national screening campaign for hypertension.

\section{Healthy eating and physical activity messages should be bound together}

The 'step-by-step route to healthy eating' also includes two steps related to the need for increasing physical activity: 'accumulate 30 minutes of physical activity daily' and 'you can do it everywhere - climb stairs, walk, dance on your own or with others'. A full account of the Brazilian Ministry of Health's strategy (Agita Brazil) to enhance the 
population level of physical activity can be found elsewhere ${ }^{6}$.

Turning information into practice: Shop Smart - the best buy

Key components. All information on healthy eating may be useless if consumers are not advised how to make the best purchases and use of their food budget. A software program called 'Shop Smart - the best buy' was produced by the Ministry of Health, in partnership with the University of Campinas, and will be available at supermarkets to help consumers make more valuable and healthier choices. It has been long believed - and this has been a main message - that the best purchases could only be made by taking advantage of the seasonal variations in fruit and vegetables prices. However, special prices and bargains are part of retailers' stock policies and the software takes all of these into account to suggest the bestvalue and nutritionally sound purchase.

Progress to date. Implementation of the Shop Smart software at points of food purchase depends upon the retailer's interest. The Ministry of Health is approaching some major supermarket chains for future partnership.

\section{Capacity-building activities: schoolteachers and bealth workers}

\section{Building the capacity of schoolteachers for nutrition promotion}

Key components. A special TV channel was established in Brazil to train elementary school teachers in a broad range of topics. This programme introduced healthy eating and good nutrition in the form of four videos (10 minutes each) that have been broadcast every two months since April 1999, potentially reaching 37 million elementary school children. All videos have the same format: their setting is an elementary school classroom with a schoolgirl and a schoolboy as anchor people. They include many playful elements, such as songs and charades, involving the children. Obesity, malnutrition, physical activity and health, food and culture, and food safety are the specific topics covered. Programmes can be watched by the teachers and/or recorded and shown to students at a later time. Support material was also produced, pre-tested and made available to teachers to help them create new classroom work with the students.

The topic - food and culture - was highly commended by the teachers and students. Its aim was to show teachers and students the cultural dimension of eating and the richness of Brazilian cultural food habits. As it is broadcast nationally, this video shows children from the different geographical regions of Brazil what their friends from other regions eat.

Informative centrefold posters were also produced in partnership with the main Brazilian magazine for schoolteachers - Nova Escola. This magazine reaches 600000 elementary school teachers throughout the country. This has proved to be a very efficient chain of communication with them, as measured by the number of phone calls and letters received for further information.

\section{Building the capacity of health workers for nutrition promotion}

Key components. Building the capacity of health workers to give advice on healthy diets and physical activity in the different stages of the life cycle is fundamental for the whole strategy's sustainability. Guidelines and manuals are being produced for the 150000 community health workers, who cover about 100 families each. In the most at-risk areas of Brazil, they cover about $75 \%$ of the population. This set of manuals includes one on 'Brazilian Regional Foods'. More than 100 edible indigenous Brazilian plants have been identified. All have been chemically analysed by different research institutions for their micronutrient contents, showing that some are extremely rich especially in vitamin A, vitamin $\mathrm{C}$ or iron. Although nutritious, most of these are being gradually lost as food sources and the purpose of this manual is to reintroduce them into people's diets. A full photographic study on these plant foods has been carried out by the Ministry of Health to be included in the manual, which should be released in November $2001^{7}$.

Diet and physical activity components were introduced in protocols used in the health services for the care of hypertensive and diabetic patients. These protocols have already been provided to about 40000 primary healthcare workers.

A national screening campaign for diabetes among individuals over 40 years of age (almost 20 million individuals were screened and $15 \%$ had high blood sugar levels) was accomplished in the first semester of this year (2001) and a new screening for hypertension will be conducted in the second semester. These screenings have two objectives: (1) to identify new cases and refer them for follow-up and (2) to raise the population's consciousness regarding these two diseases and ways to prevent them through better diet and physical activity.

\section{Innovative community-based local initiatives}

The national policies and actions described above are complemented in Brazil by a variety of state- or locally developed initiatives for nutrition promotion. These are highly innovative and creative and are fundamental for sustainable behaviour change. The federal government makes no effort to co-ordinate such activities; rather, the concept of creating networks is the norm. Local-level nutrition authorities have full autonomy to carry out their own initiatives based on technical guidelines, principles and frameworks that were built together, as previously explained. Two examples of community-based local initiatives will be presented; they refer to the City of Rio de Janeiro. 
Healthy streets by the municipality of Rio de Janeiro

Key components. A municipal law was passed in 1998 that establishes 'healthy streets' in Rio de Janeiro City. This is a good example of an initiative that can be easily expanded to other cities. This law allows the traffic to be interrupted during certain hours, every day or night, and public security is provided so that people can exercise on the streets with safety. The local community through their councils, and not the municipal authorities, choose the 'healthy streets' to be secured. Priority is given to the most deprived areas of the city, where public places for the practice of physical activity are almost non-existent.

\section{The promotion of healthful cooking among professional chefs}

Key components. Another innovative initiative in the City of Rio de Janeiro is the promotion of healthy gastronomy in some of the city's first class restaurants. The municipal health authorities, in partnership with the National Cancer Institute from the Ministry of Health, established guidelines for 'healthy meals'. These guidelines include: total calories; type and percentage of fat; percentage of meat in the total weight of the meal; amount of vegetables; low use of spices based on sodium; and cooking instructions, among others. The aim is to stimulate these restaurants to offer at least one healthy meal choice in their menus. The long-term aim is to have most of the menu choices fitting into all guidelines.

Process of achieving consensus. Healthy gastronomy guidelines were being demanded by some restaurants committed to health promotion in the city. This initiative has enabled health and tourism municipal authorities, as well as restaurant associations, to organise joint strategies to stimulate healthy gastronomy.

Progress to date. A 'Gastronomy and Health' festival is being organised by the municipality (health and tourism authorities) in partnership with the National Association of Restaurant Owners and the Restaurant Owners' Union. All restaurants interested in participating in the festival will submit at least one recipe to the organisers. This recipe has to meet all of the health guidelines mentioned previously. During a pre-defined period of time, the restaurants will offer the meal on their menu with a sign indicating that it takes part in the 'Gastronomy and Health' festival. The restaurant will give its recipe to those who choose the healthy meal and a book with all of the recipes will be published at the beginning of the festival. The municipality, in partnership with the National Association of Restaurant Owners, is producing a manual on 'Gastronomy and Health'. It is directed to all restaurants and gives details of the reasoning for each health guideline established by the municipality.

The initiative is part of a broader project called 'Healthy Rio', which is linked to the 'Agenda 21' movement. The latter was created as a follow-up of the ECO 92 meeting held in Rio and had the purpose of stimulating integrated and sustainable local development.

\section{Final remarks}

A series of innovative initiatives are taking place in Brazil at national, state and local levels to promote healthy food habits. These actions are conceptualised within a framework that has three linked areas of activity: legislation, information/communication, and capacity building. The process of formulating the National Food and Nutritional Policy, established and co-ordinated by the Ministry of Health, has created the right social environment for the launching of such initiatives by engaging all relevant social forces in a dialogue on how to improve nutritional status in the Brazilian lifestyle.

Some actions are already in place and many others need to be initiated. Although a framework and plan of action were established, as in many countries, the results actually achieved rely on opportunities sometimes provided by factors outside the nutritional field. All opportunities should be used to improve the level of governability of the nutrition plans and activities.

\section{References}

1 Monteiro CA, D'A Benicio MH, Conde WL, Popkin BM. Shifting obesity trends in Brazil. Eur. J. Clin. Nutr. 2000; 54: 342-6.

2 Monteiro CA, Conde WL, Costa Renata BL, Popkin BM. Is obesity replacing or adding to under-nutrition? Evidence from different social classes in Brazil. Public Health Nutr. 2002; 5: 105-12.

3 Ministério da Saúde. Política Nacional de Alimentação e Nutrição/Secretaria de Políticas de Saúde - DFPD, Brasilia, 2000 [Online]. Available at www.saude.gov.br. Accessed 5 September 2001.

4 Agencia Nacional de Vigilância Sanitária. Regulations on Nutrition Labelling and Serving Sizes (RDC 39, 21 March 2001) [Online]. Available at www.anvisa.gov.br. Accessed 5 September 2001.

5 Ministério da Saúde. Guias Alimentares para Crianças Menores de Dois Anos - Bases Técnicas. Brasília: Ministério da Saúde, 2001.

6 Matsudo V, Matsudo S, Andrade D, Araujo T, Andrade E, de Oliveria LC, Braggion G. Promotion of physical activity in a developing country: the Agita São Paulo experience. Public Health Nutr. 2002; 5: 253-61.

7 Ministério da Saúde. Alimentos Regionais Brasileiros. Brasília: Ministério da Saúde, 2001. 